\title{
Wireless Mesh Networks for In-Home IPTV Distribution
}

\author{
Emad Shihab, Lin Cai, Fengdan Wan, and Aaron Gulliver, University of Victoria \\ Noel Tin, Bell Canada
}

\begin{abstract}
IPTV is considered to be the next killer application. A key, challenging issue is inhome IPTV distribution with affordable deployment cost and sufficient flexibility, scalability, and reliability. In this article, we first survey and compare the emerging wired and wireless communication technologies for broadband home networks, including transmission over power lines, phone lines, coaxial cables or Ethernet cables, and IEEE $802.11 \mathrm{n}$, ultra wideband and millimeter wave wireless technologies. Considering these promising candidates for future broadband home networks, we propose three wireless mesh network architectures. These enable consumers to enjoy anywhere, anytime IPTV services without rewiring their homes. We compare the cost, reliability, and scalability of the three architectures. We further study their admission regions for IPTV, that is, the number of IPTV connections that can be supported simultaneously with satisfactory QoS. Analytical and simulation results with H.264 HDTV sources over wired, single hop wireless, and multihop wireless paths are given. These results can provide important guidelines for future broadband home network design supporting IPTV services.
\end{abstract}

nternet Protocol TV (IPTV) is considered to be the next killer application. With two-way communication capability and point-to-point distribution, each viewer can choose individual programs flexibly and at lower cost. According to a study by the Multimedia Research Group, the number of IPTV subscribers will grow from 4.3 million in 2005 to an estimated 36.8 million by 2009 , with a compound annual growth rate of 72 percent, and the IPTV service revenue will increase from $\$ 740$ million in 2005 to $\$ 4.6$ billion in 2009 , with a compound annual growth rate of 56 percent. Telecommunications service providers are racing to provide IPTV/video on demand (VoD), voice, and data — the so called triple-play services.

With fiber-to-the-x (FTTx), hybrid fiber-coaxial (HFC), and state-of-the-art digital subscriber line (DSL) technologies, high quality TV programs can be delivered to the doorstep of IPTV subscribers. However, how broadband IPTV signals eventually reach displays in different places in the home is a vital, challenging problem.

A broadband home network should be able to efficiently and effectively distribute IPTV traffic with a high level of user-perceived quality of service (QoS). Because rewiring the vast majority of existing houses with new wires such as Ethernet cables is prohibitively expensive, a better choice is to use "no-new-wires" and "no-wires" solutions. Competing communication technologies are emerging, using existing power line, phone line, coaxial cable, or wireless channels; for example, ultra wideband (UWB) - both wired and wireless - and IEEE 802.11n and millimeter-wave (mmWave) wireless technologies. They continue to reshape our vision of future broadband home networks.
To enable ubiquitous IPTV services, wireless technologies play an important role. IPTV traffic can be distributed anywhere inside and even outside the home, using single hop or multihop wireless paths. Wireless devices can discover each other using wireless broadcast beacons and form mesh networks in a plug-and-play and distributed manner. Because of auto-configuration and self-organization, wireless mesh networks can be installed by users who lack technological expertise.

According to the characteristics of different wireless technologies, we propose three different network architectures:

- Homogeneous wireless-only mesh networks

- Hierarchical heterogeneous wireless-only mesh networks

- Combined wired and wireless mesh networks

Wireless-only mesh networks and hierarchical heterogeneous wireless-only mesh networks do not have any prerequisites for current wiring in the home, so they can be deployed in existing or new homes. For heterogeneous wired and wireless mesh networks, when a wired link is available to form a reliable, high-data-rate backbone for the home network, we can use wireless technologies to further deliver IPTV traffic to the entire home ubiquitously.

To compare different architectures, the admission region (the number of IPTV connections that can be supported simultaneously with QoS guarantees) is an important measure. The following two characteristics affect the admission region. First, IPTV/VoD video compression technologies, for example - MPEG-4 H.264 - can achieve higher compression rates, but at the same time result in higher traffic burstiness (peak to average ratio) and lower error-resilience. Second, wireless channels are time-varying and location- 


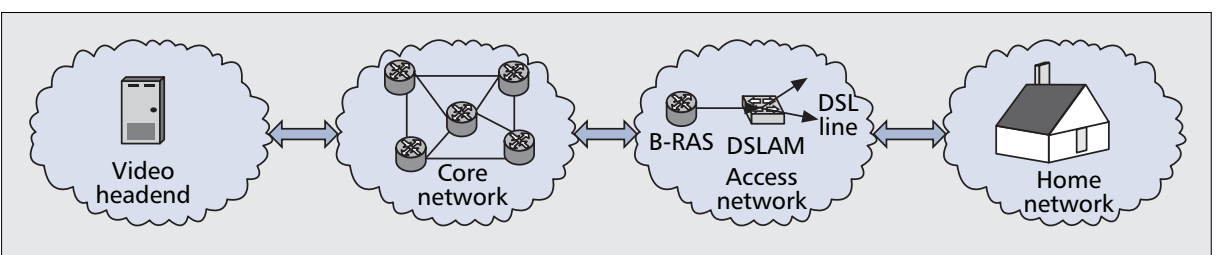

Figure 1. The IPTV system.
Ethernet - Ethernet over Category 5 cable, based on the IEEE 802.3 standard, is very successful in local area networks (LANs) because of its high data rate (up to $\mathrm{Gb} / \mathrm{s}$ ), high reliability, and wide industry support. However, Category 5 cables are not comdependent, and most wireless communication technologies adapt the data rate according to channel conditions. The achievable flow throughput over a multihop wireless path is even more dynamic.

In the following, we briefly introduce the IPTV system. We then survey the current and emerging no-new-wires and wireless communications technologies. Three wireless mesh network architectures are proposed for home networking. A fluid model-based analytical framework is used to analyze their admission regions. Extensive simulations using MPEG-4 video traces with the NS-2 network simulator were conducted to verify the analysis.

\section{The IPTV System}

A typical IPTV system consists of four main components, as shown in Fig. 1. The video headend (VH) captures all programming content, including linear programs and VoD content. The $\mathrm{VH}$ receives the content through satellite or terrestrial fiber networks. The $\mathrm{VH}$ also is responsible for encoding the video streams into MPEG-2 or MPEG-4 formats. Then, the content is encapsulated into IP packets that are sent to the core network (CN), using IP multicast or IP unicast.

The CN groups the encoded video streams into their respective channels. The $\mathrm{CN}$ is unique to the service provider and often includes equipment from multiple vendors. At this stage, IPTV traffic can be protected from other Internet data traffic to guarantee a high level of QoS.

The last-mile access network contains the broadband remote access server (BRAS), which is responsible for maintaining user policy management, such as authentication and subscription details. It also enforces QoS policies for the IPTV traffic. In the reverse direction, traffic from multiple end users is aggregated and routed to the $\mathrm{CN}$ by digital subscriber line access multiplexers (DSLAMs).

The last-meter home network distributes the data, voice, and IPTV/video traffic in subscribing homes. Each home has two to three TV sets on average. If each set shows one channel and another picture-in-picture channel, then the home network should support at least two high definition TV (HDTV) channels and two to three standard definition TV (SDTV) channels simultaneously. The average data rate for SDTV is approximately $2-5 \mathrm{Mb} / \mathrm{s}$ and for an HDTV channel, approximately $5-10 \mathrm{Mb} / \mathrm{s}$, depending on the video encoding used. For high quality video streaming services, a packet loss rate (PLR) of $10^{-6}$ or less, end-to-end latency on the order of hundreds of milliseconds, and delay jitter on the order of a few tens of milliseconds should be guaranteed.

\section{Broadband Home Network Communications Technologies \\ Wired Technologies}

Consumers have four choices for wired home networks: Ethernet cable, power line, phone line, and coaxial cable. Competing standards using these media are discussed below. mon in existing homes, and rewiring homes is too costly and inconvenient.

MoCA - Established in 2004, the Multimedia over Coaxial Alliance (MoCA) is one of the many industry-driven initiatives that promote the use of existing coaxial cables for distributing digital video and entertainment. TV sets usually are close to coaxial outlets, although on average coaxial outlets are not as widely deployed as phone and power line outlets. MoCA claimed that a throughput of $100 \mathrm{Mb} / \mathrm{s}$ is achieved by 95 percent of the coax jacks tested in the United States [5].

HomePlug AV — The HomePlug Powerline Alliance (HPPA) is promoting home power line networks and products. Power lines are the most widely available wired medium. However, power line adapters are currently more expensive than their competitors. More importantly, power lines were designed to operate at low frequencies, instead of the high frequencies required for communications [4]. Power lines suffer from high noise levels, frequency-selective and multipath fading, and interference from various appliances. Multi-phase sockets and circuit breakers also limit the connectivity. Power lines are also a shared medium after the neighborhood transformers. Therefore, power lines are not a good choice for IPTV services. The latest standard, HomePlug AV (HPAV) technology over coaxial cables, can support a raw data rate of $200 \mathrm{Mb} / \mathrm{s}$.

HomePNA - The use of phone line technologies in home networks is promoted by the Home Phoneline Networking Alliance (HPNA) [7]. Because phone lines were not originally deployed for delivering TV signals, most phone line outlets are not located within easy reach of suitable TV locations. In addition, the physical medium, twisted-pair or flat wires, has a much smaller bandwidth than shielded coaxial cables, and the connection topology, daisy-chain, is likely to introduce noise and interference. Therefore, newer HPNA standards use both the phone lines and coaxial cables. With the HPNA 3.0 standard, a maximum raw data rate of $128 \mathrm{Mb} / \mathrm{s}$ (extensible to 240 $\mathrm{Mb} / \mathrm{s}$ ) can be achieved.

Preliminary test results of MoCA, HPAV, and HPNA 3.0 adapters over cable lines show that they can achieve a linklayer throughput of $80-100 \mathrm{Mb} / \mathrm{s}$.

\section{Wireless Technologies}

Emerging wireless technologies can achieve data rates comparable to or even higher than those of wired competitors.

IEEE 802.1 $1 n$ - The IEEE 802.11n standard specifies the communications in wireless local area networks (WLANs) in the 2.4 and $5 \mathrm{GHz}$ license-free bands. Raw data rate of up to $540 \mathrm{Mb} / \mathrm{s}$ can be achieved within a $50 \mathrm{~m}$ range. The standard also incorporates the security and QoS enhancements specisupporting heterogeneous traffic in both indoor and outdoor environments. With a transmission range of $50 \mathrm{~m}$, a single access point (AP) can cover an entire house in most cases. However, this high transmission range also leads to significant interference among neighbors. In addition, there are many other wireless and electronic devices operating in the same fied in the IEEE 802.11e standard. This makes it suitable for 


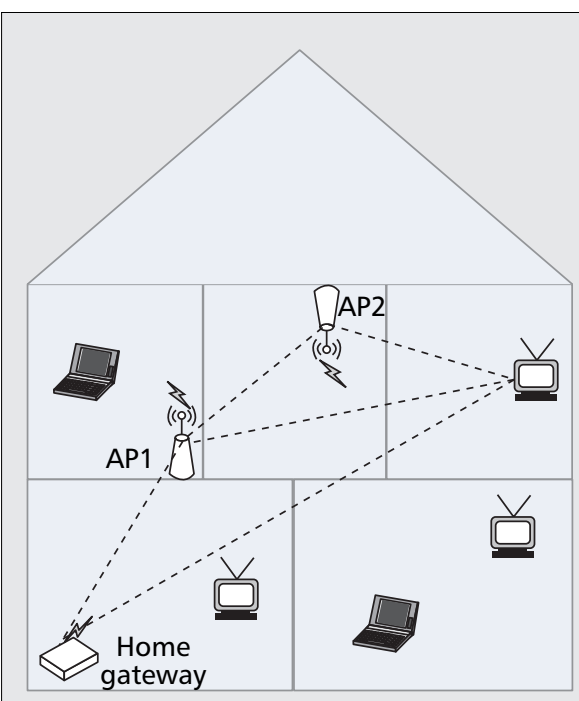

(a)

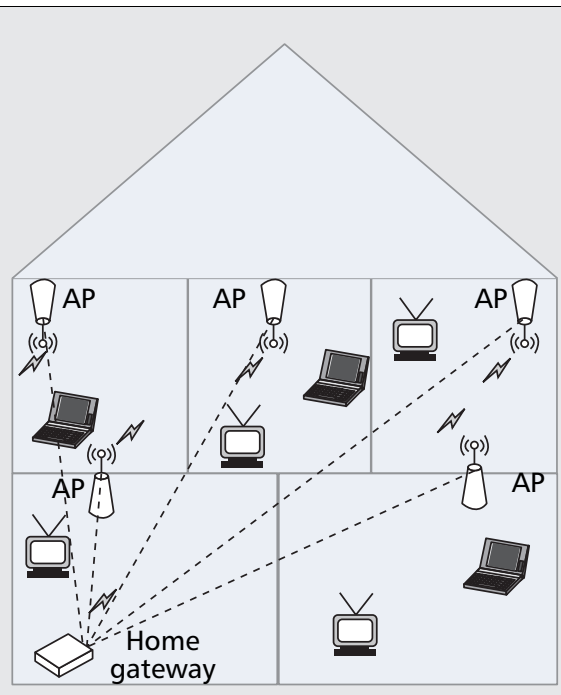

(b)

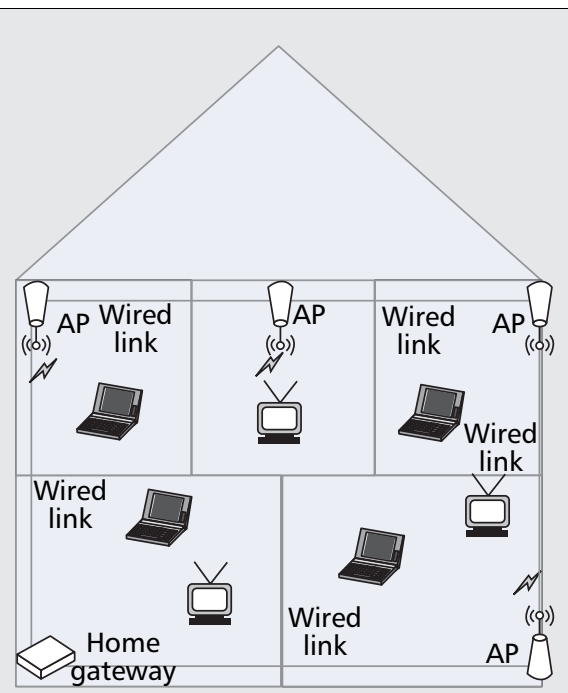

(c)

Figure 2. Home mesh network architectures: a) homogeneous wireless only; b) hierarchical heterogeneous wireless; c) heterogeneous wired and wireless.

bands, for example, microwave ovens and hands-free headsets, which results in unpredictable interference to $802.11 \mathrm{n}$ transmissions.

Ultra Wideband - UWB refers to any radio technology having a bandwidth exceeding the lesser of $500 \mathrm{MHz}$ or 20 percent of the center frequency [8]. The Federal Communications Commission (FCC) allows UWB transmissions over the 3.1 to $10.6 \mathrm{GHz}$ wireless band, with a stringent power spectral density limit of $-41.3 \mathrm{dBm} / \mathrm{MHz}$. The raw data rate varies from $55 \mathrm{Mb} / \mathrm{s}$ to $480 \mathrm{Mb} / \mathrm{s}$, within a range of $10 \mathrm{~m}$. There are two competing UWB technologies: direct sequence (DS) UWB, supported by the UWB forum, and multiband orthogonal frequency-division multiplexing (OFDM) UWB, supported by the WiMedia Alliance. Recently, OFDM-based UWB communications over coaxial cables also was proposed.

The low power and short range characteristics of UWB wireless communications is ideal for the home environment because it does not significantly affect other narrowband communications. In addition, service providers do not want their broadband signals to be accessible to other (non-subscribing) homes, so the short transmission range and high attenuation by barriers such as concrete walls are desirable characteristics from the viewpoint of a service provider. On the other hand, due to the limited transmission range, more APs are required to cover an entire house, which results in higher cost.

Millimeter Wave (mmWave) - The FCC also has approved license-free use of the $57-64 \mathrm{GHz}$ mmWave band at a transmission power of up to $40 \mathrm{dBm}$ effective isotropic radiated power (EIRP), which is significantly higher than the limits in other WLAN and wireless personal area network (WPAN) standards. The IEEE 802.15.3c task group was formed in March 2005 to develop a mmWave-based alternative physical (PHY) layer for WPANs. Prototypes using mmWave to achieve a raw data rate up to $2 \mathrm{~Gb} / \mathrm{s}$ within a 1-2 m range have been developed [10]. At these high frequencies, mmWave signals suffer from high oxygen and water vapor absorption, and they cannot penetrate through solid materials (like walls). Therefore, each room may require a separate AP, with negligible interference between transceivers in different rooms.

\section{Mesh Home Network Architecture}

Considering the heterogeneity of different wireless technologies, we propose three home network architectures for ubiquitous in-home IPTV distribution:

- Homogeneous wireless-only mesh network

- Hierarchical wireless-only mesh network

- Combined wired and wireless mesh network

\section{Homogeneous Wireless-only Mesh Networks}

A homogeneous wireless-only mesh network consists of several APs that communicate with each other and connect to mobile or stationary client devices using the same wireless technology, for example, UWB, as shown in Fig. 2a. These APs in a single home are assumed to be in the same collision domain; thus, they should share the wireless resources using random access technologies in a distributed manner, or they can choose a centralized controller to schedule collision-free transmissions. The mesh structure has the advantage of high reliability, error resilience, and easy installation. By deploying more APs than necessary, we can eliminate dead zones and reduce network faults due to wireless channel impairments or other failures [3]. However, to ensure the stringent QoS for IPTV traffic, routing and scheduling becomes complicated.

\section{Hierarchical Heterogeneous Wireless-only Mesh Networks}

The second approach is to build hierarchical wireless networks, using heterogeneous radio technologies, as shown in Fig. 2b. For example, because $802.11 \mathrm{n}$ has sufficient transmission range, we can use an IEEE 802.11 n router (home gateway) to distribute traffic to several APs in a single hop. The 802.11 router and the APs construct the wireless backbone of the home network, and the $802.11 \mathrm{n}$ router can be the centralized controller of the backbone. An AP and the nearby wireless devices form a piconet, and the AP further distributes the traffic to all devices in the piconet in a single hop using another wireless technology, for example, mmWave. The APs are equipped with both an IEEE $802.11 \mathrm{n}$ radio and a mmWave radio, and each controls a piconet. Because the interference between different wireless technologies operating in different frequency bands is negligible, this architecture can simultaneously utilize the wireless resources in both bands. The installa- 
tion, QoS-aware routing, and scheduling of the hierarchical mesh network are comparatively simple because the IPTV traffic can be relayed to any device in two hops, and these two hops use different radios so there is no interference between them. However, APs with dual-radios are more costly. Furthermore, the network is vulnerable to failures in the upper level, which might lead to service interruption of the entire home. Unfortunately, $802.11 \mathrm{n}$ is susceptible to interference from other WLANs and electronic devices, and the other high-data-rate wireless technologies (UWB and mmWave) may not cover the entire house in a single hop.

\section{Heterogeneous Wired and Wireless Mesh Networks}

As shown in Fig. 2c, using a combination of wireless and wired distribution technologies is a promising, no-new-wires solution. Existing wired links, for example, coaxial cables or phone lines, construct the backbone of the home network. A few wireless APs, serving as piconet controllers, further relay the IPTV traffic to any place in the home, using high-datarate wireless technologies, for example, UWB or mmWave. Using this architecture, the home network is scalable and simple to setup. Because it relies on wireless technologies with a small transmission range and low interference, the spatial capacity of the home network is large. In addition, the wired backbone is reliable in general, and a failure in the wireless network only affects a small area, not the entire home network.

\section{Performance Evaluation}

Given the different network architectures, one immediate question is how many IPTV connections can be supported simultaneously with guaranteed QoS, or what are the admission regions? The answer not only helps service providers and consumers to choose the best technologies for home networking, but also provides important guidelines for planning the entire IPTV system.

\section{System Model}

To support IPTV traffic with stringent QoS requirements, we assume that there is a centralized controller in each network with a shared medium (the wired backbone, the wireless piconet, or the wireless mesh networks), which schedules nonoverlapping channel times for collision-free transmissions and selects routes appropriately.

Because wireless channel conditions are time-varying, most wide-band wireless technologies can adapt transmission data rates according to channel conditions or the received signalto-noise ratio (SNR). For instance, the over-the-air raw data rate of $802.11 \mathrm{n}$ varies from $100 \mathrm{Mb} / \mathrm{s}$ to $540 \mathrm{Mb} / \mathrm{s}$, and that of UWB varies from around $55 \mathrm{Mb} / \mathrm{s}$ to $480 \mathrm{Mb} / \mathrm{s}$. With physicallayer adaptive-rate control and link-layer retransmissions, packet losses due to transmission errors are negligible; however, the link throughput (achievable data rate) becomes timevarying. Finite state Markov chains have been widely adopted as packet-level wireless channel models because they are reasonably accurate in capturing wireless channel variations [9]. We developed a finite state Markov chain for a single hop wireless channel as follows: each state corresponds to a different SNR range and thus a different data rate, and the statetransition probabilities are chosen according to the time-correlation of the wireless channel. Similarly, a Markov chain can be developed for a multihop wireless path: each state corresponds to certain SNR ranges for each hop [6]. We use the Markov chain to model the packet-level wireless channel behavior for both the analysis and simulations.

In a homogeneous wireless-only mesh network, the IPTV traffic can be delivered to a TV in multiple wireless hops. All packets to be scheduled are stored in the buffer for the first hop. A packet over multiple hops will be scheduled with nonoverlapping channel times for each hop. Each hop may choose a different transmission rate and thus requires a different channel time. Longer hops usually have a lower SNR and thus a lower data rate, but fewer hops are required. On the other hand, the ideal locations for APs, for example, in the middle of a house, may not be accessible. There is an inherent trade-off in determining the number of hops and the optimal locations of APs [11]. As shown in Fig. 2a, IPTV signals from the home gateway can reach the TV in one hop, via a two-hop path relayed by AP1, or via a three-hop path relayed by both AP1 and AP2. Thus, we must investigate the different paths and choose the best one. To obtain a lower bound on the admission region, all IPTV traffic is routed to the device in the worst location, using the best path.

For a hierarchical heterogeneous wireless mesh network, we consider two hierarchical levels. Because wireless technologies with a larger transmission range normally have lower transmission rates, the bottleneck with this architecture is in the higher level, the one-hop wireless backbone. To simplify the analysis, we also assume that the channel qualities from the gateway to all APs are the same as that of the worst one. Then, the resulting admission region of the one-hop wireless network will be a lower bound.

For heterogeneous wired and wireless mesh home networks, we should investigate the capacities of both the wired backbone network and the wireless mesh network. The smaller capacity determines the admission region of the entire network.

\section{Analytical Framework}

To quantify the maximum number of video sources that can be supported with satisfactory QoS, we should investigate the admission regions of the wired backbone, wireless backbone, and multihop wireless paths. Then, we can identify the bottleneck and obtain the admission region of the home network. Here, we briefly introduce the analytical framework; the detailed mathematical derivations can be found in [6].

IPTV is sensitive to end-to-end delay, jitter, and loss rate. Delay jitter can be absorbed using the receiver buffer. Packets suffering excessive delay for real-time TV programs are considered useless and will be discarded by the receiver, so the end-to-end delay should be bounded. With bounded delay, packet losses are mainly due to buffer overflow. The home network can be allocated a portion of the end-to-end delay and loss budget. Given the delay budget, we can determine the maximum queuing delay in a home network, and the buffer size of the bottleneck can be determined accordingly. Then, we should derive the packet loss probability given the video traffic model and buffer size.

The fluid flow model [2] was used to model variable bit rate video sources, which approximates the video packet arrival process as a continuous flow of information. Thus, some existing mathematical tools for continuous variables can be applied. One video source is modeled as the multiplexing of a number of mini on/off sources. Each mini source independently alternates between an off state and an on state, where a constant rate of data is generated during the on state. The state-transition probabilities and the data rate of each mini-source in the on state are chosen according to the mean, variance, and autocovariance of the original video source. A number of video sources can be modeled as a continuous-time Markov chain, in which state $i$ represents that $i$ mini-sources are in the on state.

For a wired link with fixed data rate, given the video source 


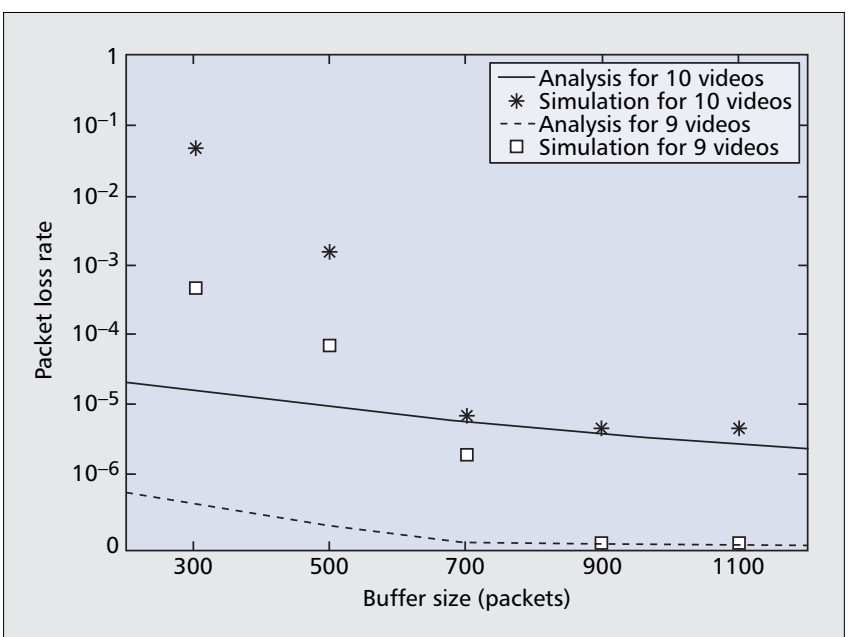

Figure 3. PLR vs. buffer size for a wired link.

model and buffer size, the queue length distribution and the probability of packet loss due to buffer overflow can be derived [2]. In the wireless domain, we also use finite state Markov chains to model the variable data rates of single hop and multihop wireless paths, respectively. The Markov model of the video sources and that of the wireless paths can be combined together mathematically, so the queuing delay distribution and packet loss probability due to buffer overflow can be derived as functions of the number of video sources and the buffer size. Then, we can obtain the admission regions of both the wired and wireless networks with a guaranteed loss rate and delay jitter [6].

\section{Performance Evaluation by Simulation}

Extensive simulations were performed using the NS-2 network simulator. The H.264 video trace of "From Mars to China" in HDTV format $(1920 \times 1080 i)$ was used [1]. Its average bit rate is $4.85 \mathrm{Mb} / \mathrm{s}$, the variance is $5.82 \times 10^{9}$, the autocorrelation decay coefficient is 0.215 per second, and the frame rate is 30 frames/sec. The video frames ( $I, B, P$ frames) are fragmented into User Datagram Protocol (UDP) packets of 1000 bytes. In the simulation, each video source traverses a $100 \mathrm{Mb} / \mathrm{s}$ wired link, and all sources are multiplexed in the bottleneck with limited buffer size. The starting times of the video sources are randomized, and the first 30 seconds of the simulation results are not counted. For each parameter setting, the average of ten 30-minute long simulations with different random seeds is used to capture loss rates as low as $10^{-6}$.

Figure 3 shows the analytical and simulation results of the PLR versus the buffer size of the bottleneck in a wired link with a constant data rate of $85 \mathrm{Mb} / \mathrm{s}$. As shown in the figure, the loss rate decreases sharply with respect to the buffer size when the buffer size is less than 700 packets and then flattens out. This is because a single $I$ or $P$ frame of the video source can be more than 300 kilobytes, and thus we require a buffer size of $300+$ packets to accommodate a single frame. From the results, a buffer size of no less than 700 packets is desirable to keep the PLR low. Conversely, we can use packet shaping technologies to reduce the burstiness of the traffic. Another observation is that the fluid model provides a good approximation only when the buffer size is large $(\geq 700)$. Because the analytical model assumes that packets arrive at a constant rate during the on state, the model cannot capture the traffic burstiness during an on period. Both the analytical and simulation results show that, to ensure a PLR less than $10^{-6}$, the maximum number of video sources that can be supported is nine. If ten sources are admitted, the PLR is always larger than $10^{-6}$, even with a buffer size larger than 1100 packets.

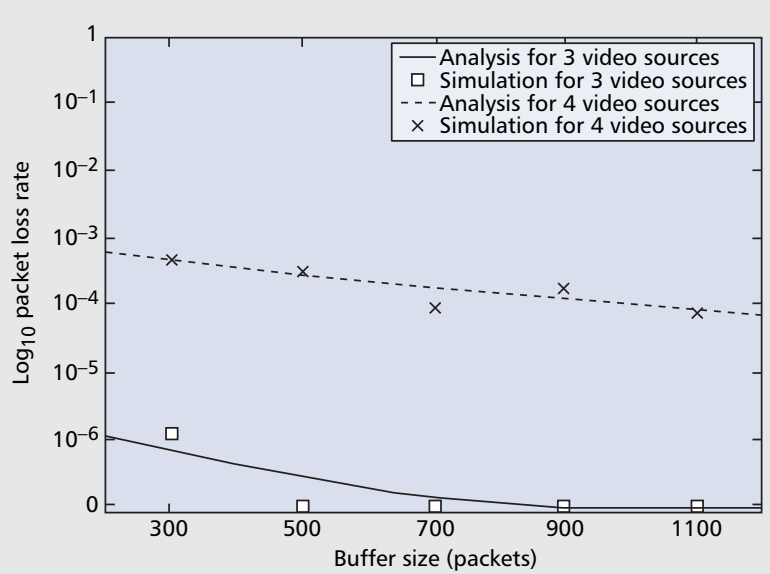

(a)

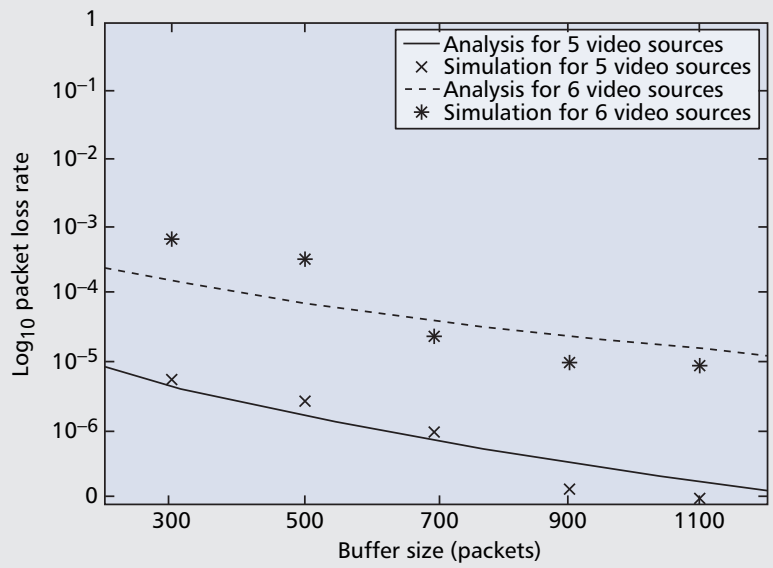

(b)

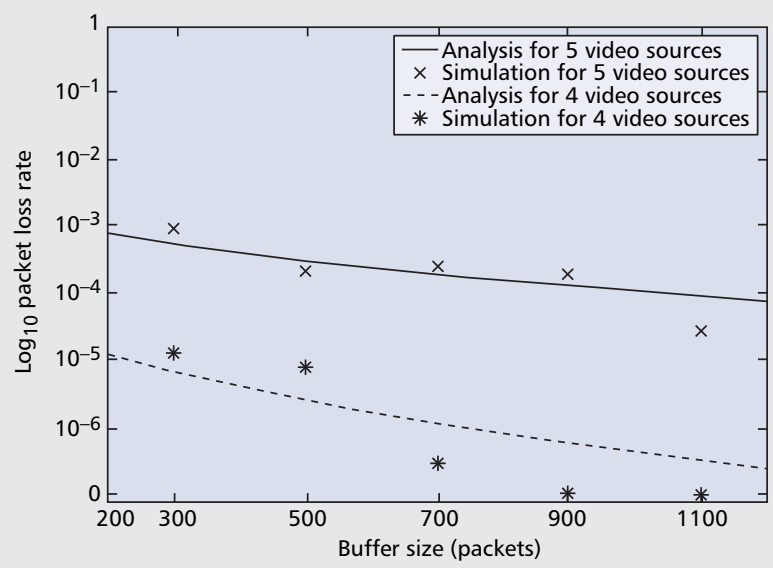

(c)

Figure 4. PLR vs. buffer size for multihop wireless paths: a) single-hop; b) two-hop c) three-hop.

We repeated the simulation using a wireless link with a rate changing between $90 \mathrm{Mb} / \mathrm{s}, 65 \mathrm{Mb} / \mathrm{s}$, and $40 \mathrm{Mb} / \mathrm{s}$ according to a Markov chain, but maintaining an average rate of $85 \mathrm{Mb} / \mathrm{s}$. Due to space limitation, the results are not shown here. The results demonstrate that, although the wireless link has the same average data rate as the wired one, only five video sources can be supported, which is far below the admission region of the wired link. Therefore, if we use only the average 
data rate of a time-varying link to calculate the admission region, the results will be too optimistic.

Next, we investigate multihop wireless mesh networks. We use the topology shown in Fig. 2a and investigate the performance of the single hop (gateway to TV), two-hop (relayed by AP1), and three-hop (relayed by both AP1 and AP2) paths.

We use the data rates of Tzero UWB devices (using the WiMedia standard) as an example. The raw per-hop data rates are $53.3,80,106.7,160,200$, and $480 \mathrm{Mb} / \mathrm{s}$, and the corresponding achievable data rates are 37.5, 53.3, 64.4, 83.1, 99.2 and $139 \mathrm{Mb} / \mathrm{s}$, respectively. The gap between the raw and achievable data rates is larger when the data rate is higher, because the physical and link layer headers are always transmitted at the lowest data rate. For the longest hop between the home gateway and the TV, the received signal strength and the achievable data rate are low. The typical achievable data rate is $37.5 \mathrm{Mb} / \mathrm{s}$ (in State 1 ), but occasionally can reach $53.3 \mathrm{Mb} / \mathrm{s}$ (in State 2). Denote $P_{i j}$ as the state transition probability from state $i$ to state $j$. We set $P_{12}=0.01$ and $P_{21}=0.5$. For the hop between AP1 and the TV, the channel has three states, with achievable data rates 64.1 (in State 1), 83.1 (in State 2), and $99.2 \mathrm{Mb} / \mathrm{s}$ (in State 3 ). $P_{11}=1-P_{12}=0.1, P_{21}$ $=P_{22}=\left(1-P_{23}\right) / 2=0.1$, and $P_{32}=1-P_{33}=0.1$. For the shortest hops between the home gateway, AP1, and AP2, the achievable data rates are 83.1 (in State 1), 99.2 (in State 2), and $139 \mathrm{Mb} / \mathrm{s}$ (in State 3$) . P_{11}=1-P_{12}=0.1, P_{21}=P_{22}=(1$ $\left.-P_{23}\right) / 2=0.05$, and $P_{32}=1-P_{33}=0.05$. For a packet over a multihop path, the requested channel time equals the sum of the channel times over each hop (i.e., packet size over the achievable data rate of each hop), and all other overheads such as the beacon time are ignored.

Figure 4 shows the admission regions of the one-hop, two-hop, and three-hop wireless paths. For the single hop case, only three video sources can be supported due to the low data rate. Comparing the two-hop and three-hop cases, two hops can support six video sources, whereas three hops can support only five. Although the per-hop data rate of the three-hop case is higher, the overall channel time required is slightly higher than that of the two-hop case because each packet must be transmitted three times. In addition, the variation of the wireless path with more hops is higher, which also affects the PLR. According to the analytical and simulation results, the best choice with this topology is to use AP1 only as a relay, resulting in an admission region of five.

\section{Conclusions}

Consumers will subscribe to IPTV services that provide satisfactory QoS, value, service differentiation, and convenience. With emerging high-data-rate wireless technologies, wireless mesh home networks can provide personalized IPTV services anytime, anywhere, and without house rewiring. This is a key differentiation and convenience that consumers are willing to pay for. An easy-to-install wireless home network also can save significant truck-roll costs and ensure the profitability of new broadband services, so it is vital for the success of IPTV. Considering the characteristics of different wireless and wired technologies, we have proposed three wireless mesh network architectures and studied their admission regions for IPTV services. These results can provide important guidelines for future broadband home networks.

\section{Acknowledgment}

This work has been supported in part by a research grant from Bell University Laboratories (BUL) under the sponsorship of Bell Canada and an NSERC CRD grant.

\section{References}

[1] P. Seeling and M. Reisslein, "Evaluating Multimedia Networking Mechanisms Using Video Traces," IEEE Potentials, vol. 24, no. 4, Oct. -Nov. 2005, pp. 21-25.

[2] B. Maglaris et al., "Performance Models of Statistical Multiplexing in Packet Video Communications," IEEE Trans. Commun., vol. 36, no. 7, July 1988, pp. 834-44.

[3] I. Akyildiz, X. Wang, and W. Wang, "Wireless Mesh Networks: A Survey," Computer Networks, vol. 47, 2005, pp. 445-87.

[4] Y. Lin et al., "A Power Line Communication Network Infrastructure for the Smart Home," IEEE Wireless Commun., vol. 9, no. 6, Dec. 2002, pp. 104-11

[5] "Multimedia over Coax Alliance Field Test Report Executive Summary," Tech. rep. June 2005

[6] E. Shihab et al., "Performance Analysis of IPTV in Home Networks," Proc. IEEE GLOBECOM, Washington, DC, Nov.-Dec. 2007.

[7] The HomePNA Alliance, http://www. homepna.org

[8] W. Zhuang, X. Shen, and Q. Bi, "Ultra-Wideband Wireless Communications," Wireless Commun. and Mobile Comp., Special Issue on Ultra-Broadband Wireless Communications for the Future, vol. 3, no. 6, 2003, pp. 663-85.

[9] H. S. Wang and N. Moayeri, "Finite-State Markov Channel - A Useful Model for Radio Communication Channels," IEEE Trans. Vehic. Tech., vol. 44, no. 1, Feb. 1995, pp. 163-71

[10] S. Reynolds et al., "A Silicon $60 \mathrm{GHz}$ receiver and Transmitter Chipset for Broadband Communications," IEEE J. Solid-State Circuits, vol. 41, no. 12 Dec. 2006 , pp. $2820-30$

[11] D. Li and J. Pan, "Evaluating MPEG-4 AVC Video Streaming over IEEE 802.11 Wireless Distribution System," Proc. WCNC, Las Vegas, NV Mar.-Apr. 2008.

\section{Biographies}

EMAD SHIHAB (emads@ece.uvic.ca) received his B.Eng. (with distinction) from the University of Victoria, Canada, in 2007. He is currently pursuing his M.A.Sc. in electrical and computer engineering at the same university. He has held various coop positions at Research In Motion, Waterloo, Ontario, Canada, and Telus Communication Inc., Vancouver, British Columbia, Canada. His research spans several areas in wireless communications and networking, with special interests in distribution technologies, network architectures, protocol design, capacity analysis, and resource management of broadband wireless and wired networks.

LIN CAl [S'00, M'06] (cai@ece.uvic.ca) received her M.A.Sc. and Ph.D. degrees (with an Outstanding Achievement in Graduate Studies Award) in electrical and computer engineering from the University of Waterloo in 2002 and 2005 respectively. Since July 2005 she has been an assistant professor in the Department of Electrical and Computer Engineering at the University of Victoria. Her research interests focus on network protocol and architecture design supporting emerging multimedia traffic over wireless, mobile, ad hoc, and sensor networks. She serves as Associate Editor for IEEE Transactions on Vehicular Technology (2007-), EURASIP Journal on Wireless Communications and Networking (2006-), and International Journal of Sensor Networks (2006-).

FENGDAN WAN (fdwan@ece.uvic.ca) received her B.Eng. from Southeast University, Nanjing, China, in 2005. She is currently pursuing her M.A.Sc. in electrical and computer engineering at the University of Victoria. Her recent research interests are in the area of wired/wireless networking; including resource management in heterogeneous wired/wireless networks, end-to-end QoS provisioning and performance analysis.

T. AARON GULLIVER [SM] (agullive@ece.uvic.ca) received a Ph.D. degree in elec trical engineering from the University of Victoria in 1989. He joined the University of Victoria in 1999 and is a professor in the Department of Electrical and Computer Engineering. His research interests include information theory; coding theory; cryptography; iterative codes and decoding; wireless, ad hoc, and senso networks; cognitive radio; MIMO systems and space-time coding; and ultra wideband communications. From 1989 to 1991 he was employed as a defense scientist at the Defense Research Establishment, Ottawa, Canada. He has held academic positions at Carleton University, Ottawa, Canada, and the University of Canterbury, Christchurch, New Zealand. He is a member of the Association of Professional Engineers and Geoscientists of British Columbia, Canada. In 2002 he became a Fellow of the Engineering Institute of Canada. He was registration chair for the 1995 IEEE International Symposium on Information Theory, which was held in Whistler, Canada. In 2001 and 2005 he was co-chair of the IEEE Pacific Rim Conference on Communications, Computers, and Signal Processing. He was also co-chair of the 2003 IEEE Information Theory Workshop held in Paris, France. He has been on the organizing committees of numerous other international conferences. He is currently an editor for IEEE Transactions on Wireless Communications. From 2000 to 2003 he was Secretary and a member of the Board of Governors of the IEEE Information Theory Society.

NOEL TIN [M'O0] received B.Sc., B.A., and M.Sc. degrees from Queen's University, Kingston, Ontario, Canada in 1998 and 2000, respectively. He has been with Bell Canada and Bell Mobility since 2000, working as a network architect, technology advisor, and most recently as a senior network architect. His research interests linclude wireless and wireline acess technology, home networking, smart antenna, wireless and xDSL devices, and remote device management. 